\section{Pavlo Sivolapov, Oleksiy Myronyuk, Denys Baklan, Taras Berehovyj}

\title{
FORMATION OF EFFECTIVE CONCENTRATION OF FILM FORMING SUPERHYDROPHOBIC COATINGS BASED ON SILICON DIOXIDE
}

The object of research is the hydrophobization of a coating based on styrene-acrylic copolymer and cellulose acetate butyrate with pyrogenic silicon dioxide. The hydrophobicity or hydrophilicity of a surface depends mainly on the chemical structure of the surface and its roughness. A relief hydrophilic surface will be wetted much more easily than a flat surface of the same material, and vice versa - an increase in the roughness of a hydrophobe is accompanied by a more pronounced repulsion of water. The size and number of irregularities significantly affect the wetting characteristics of the investigated surface. The combination of low surface energy and relief structure forms a superhydrophobic surface. Such surfaces have found application in self-cleaning, frost-resistant, antifriction, electrically conductive and oil-sorbing coatings. In this work, styrene-acrylic copolymer and cellulose acetate butyrate were used as a film former. Pyrogenic silicon dioxide Aerosil $R$ 972, which was hydrophobized with dimethyldichlorosilane, was used as a nanofiller. In this work, the compositions were applied to laboratory glasses by dip coating. The determination of surface energy was carried out using glycerin and diiodomethane. The hydrophobic properties of the coatings were evaluated by measuring the contact angles with water using a goniometer. The analysis of the morphological structure of the coating surface with photographs of a scanning electron microscope has been carried out. The energy state of the surface of the created superhydrophobic coatings has been determined and their surface energies have been calculated using the technique based on the Owens-Wendt model. The process of hydrophobization of polymer-based coatings is investigated, which occurs both due to chemical modification with the introduction of silicon dioxide and due to the creation of nanoroughness of the surface layer of the coatings. The dependences of this process on materials are investigated using an electron microscope and the determination of their surface energy depending on the $\mathrm{SiO}_{2}$ filling. The values of the critical concentration of the modifier for the transition of polymers to the superhydrophobic state have been determined. As a result, the influence of polymer crystallinity on hydrophobization was determined by comparing changes in the surface energy of materials during their modification.

Keywords: superhydrophobic coatings, fumed silica, Owens-Wendt method, surface energy, water-repellent surfaces.

\section{How to cite}

Sivolapoz, P., Myronyuk, O., Baklan, D., Berehovyj, T. (2021). Formation of effective concentration of film forming superhydrophobic coatings based on silicon dioxide. Technology Audit and Production Reserves, 3 (3 (59)), 6-9. doi: http://doi.org/10.15587/2706-5448.2021.233535

\section{Introduction}

The ability of solid surfaces to repel certain liquids depends mainly on two factors: the chemical composition of the active groups on the surface and its morphology. So, materials on the surface of which non-polar chemical groups predominate (for example $-\mathrm{CH}_{2},-\mathrm{CH}_{3},-\mathrm{CHal}$ ) have a low surface energy (35-40 MJ $/ \mathrm{m}^{2}$ in carbon [1]). This means that they are not able to create strong bonds with the liquid and show hydrophobicity (the angle of wetting of carbon is approximately $90^{\circ}$ [1]). While bodies with a polar surface, for example, metal oxides, exhibit high surface energy, which can reach $1000 \mathrm{MJ} / \mathrm{m}^{2}$, for example, in $\mathrm{CaO}$, the surface energy is $1032 \mathrm{MJ} / \mathrm{m}^{2}$ [2].

The surface structure also has a significant effect on wetting: as a rule, the presence of irregularities on the surface significantly increases the output wetting ability [3, 4]. That is, a relief hydrophilic surface will be wetted much easier than a flat surface of the same material, and vice versa - an increase in roughness by a hydrophobe is accompanied by a more pronounced repulsion of water. This is because the appearance of roughness on a flat surface leads to an increase in its wetting angle and an increase in adhesion between this surface and the liquid, since the contact area on an uneven plane increases [5]. The size and number of irregularities to a large extent affect the characteristics of wetting of the investigated surface.

As a result of the combination of the low energy of the surface with its relief structure, a superhydrophobic surface is formed. One of two types of wetting is realized for them [6]: - homogeneous, in which the liquid contacts the entire surface of the solid, completely filling all its irregularities (the state of the monogram [7]); 
- heterogeneous, in which air is entrained inside the surface irregularities (the Cassi state [8]).

In addition to the named basic models of wetting, there are also a number of other models that describe states intermediate between monograms and Cassi [9, 10].

Such surfaces have very strong water-repellent properties, therefore coatings capable of forming superhydrophobic surfaces are often used to give materials the following characteristics:

- self-cleaning [11];

- frost-resistant [12];

- anti-friction [13];

- electrically conductive [14];

- oil-sorbing [15].

In the case of using organomineral dispersions for the formation of textured surfaces based on thin-layer coatings, fillers are the main means of forming the relief. Accordingly, predicting and regulating the effective filler/matrix ratio is an urgent problem.

Thus, the object of research is the hydrophobization of coatings based on styrene-acrylic copolymer and cellulose acetate-butyrate with pyrogenic silicon dioxide. Accordingly, the aim of research is to establish the regularities of the formation of the values of the effective concentration of the filler to achieve the superhydrophobic state of such systems.

\section{Methods of research}

2.1. Materials. In this work, the following polymer film-formers were used as a binder: styrene-acrylic copolymer Pliolite AC-80 (Omnova solutions, USA) and cellulose acetate butyrate CAB-381-0.5 (Eastman, USA). The mass fractions of acetate and butyrate groups are 13.5 and 37 mass. \%, according. Pyrogenic silicon dioxide Aerosil R 972 (Evonik, Germany), hydrophobized with dimethyldichlorosilane, was used as a nanofiller. The xylene and tetrahydrofuran solvents were purchased from VWR International (USA). Glycerin and diiodomethane (VWR International, USA) were test liquids for determining the surface energy of the coatings. Levenhuk G50 laboratory glasses (USA) were used as substrates for coatings.

2.2. Technique for the preparation and analysis of superhydrophobic coatings. The superhydrophobic coatings were based on prepared $5 \%$ solutions of AC-80 polymers and cellulose acetobutyrate in xylene and tetrahydrofuran, respectively. To introduce particles of silicon dioxide into the polymer composition, a dispersion was prepared with $4 \mathrm{~g}$ of Aerosil, which was used to disperse $26.6 \mathrm{ml}$ of the solvent by ultrasound for $10 \mathrm{~min}$. The resulting dispersion was mixed on a magnetic stirrer at a speed of $600 \mathrm{rpm}$ for $15 \mathrm{~min}$, after which it was mixed with a polymer solution in the required ratios, and brought to the finished state in the same way. After mixing, the compositions were applied to laboratory glasses by dip coating. The applied coatings were dried at room temperature for 24 hours.

The hydrophobic properties of the obtained coatings were estimated from the results of measuring the contact angles of wetting with water $\theta$ (WCA). For these measurements, a Kyowa DM-701 goniometer (Japan) was used together with the WinDrop software. On each sample, 5 measurements of the contact angle were made, and the arithmetic mean of 5 measurements of each of the three samples of the studied formulation was considered the final result. The measurement error was $\pm 5 \%$.

The analysis of the morphological structure of the surface of the obtained coatings was carried out using photographs of a scanning electron microscope (SEM) Quanta 650 FEG (FEI, USA).

To characterize the energy state of the surface of the created superhydrophobic coatings, their surface energies $\sigma$, $\sigma_{L}^{D}$ and $\sigma_{L}^{P}$ were calculated using the method based on the Owens-Wendt model [16] described in [17].

The value of surface tension and its components $\sigma_{L}^{D}$ and $\sigma_{L}^{P}$ test fluids are given in Table 1 .

Table 1

Surface tension and its components of test liquids [18]

\begin{tabular}{|c|c|c|c|}
\hline \multirow{2}{*}{ Liquid } & \multicolumn{3}{|c|}{ Surface tension, $\mathrm{mJ} / \mathrm{m}^{2}$} \\
\cline { 2 - 4 } & total value & dispersion component & polar component \\
\hline Diiodomethane & 50.8 & 50.8 & 0 \\
\hline Glycerol & 64 & 34 & 30 \\
\hline Water & 72.8 & 21.8 & 51 \\
\hline
\end{tabular}

For a more accurate determination of the surface energy, liquids with different polarities were used. As it is possible to see from the Table 1, diiodomethane acts as a non-polar liquid, water is polar, and glycerol has approximately the same values of both the polar and dispersion components of the surface tension.

\section{Research results and discussion}

In order to determine the minimum amount of filler required to achieve a superhydrophobic state of the coating, two rows of coatings were prepared, one for each polymer. The concentration of silica particles in coatings based on cellulose acetate butyrate (CAB) ranged from 0 to 30 wt. \% in steps of 5 wt. \% In relation to the mass fraction of the polymer. In the case of styrene-acrylic (AC-80), the concentration increased from 0 to 6 wt. \% in steps of 1 wt. \%. To reduce the experimental error, 3 samples were prepared for each value of the filler concentration. The assessment of the hydrophobicity of the coatings was carried out according to the results of measurements of the contact angle of wetting with water. The results of these measurements are presented in the form of graphs in Fig. 1. The contact angles of pure polymer films of cellulose butyrate acetate and styrene-acryl without the addition of filler were $72^{\circ}$ and $78^{\circ}$, respectively, which is in good agreement with the values of similar polymers in previous studies [19, 20].

As can be seen in the graphs (Fig. 1), with an increase in the content of the filler in the coating composition, the contact angle with water increases to reach a value of $125-130^{\circ}$. The minimum critical concentration of polymers is $6 \mathrm{wt}$ \% In the case of styrene-acrylic and $30 \mathrm{wt}$. \% for cellulose acetate butyrate. With the content of such an amount of filler in the composition of the coating compositions, the effect of superhydrophobicity was achieved due to:

1) decrease in the surface energy of coatings due to the chemical modification of silicon dioxide with dimethyldichlorosilane;

2) appearance of nanoroughness in the surface structure of the resulting films. 
For a deeper understanding of the surface transformations that accompany the hydrophobization of coatings, their surface energy was calculated using the Owens-Wendt method. The calculation results are presented in the form of a graph in Fig. 2.
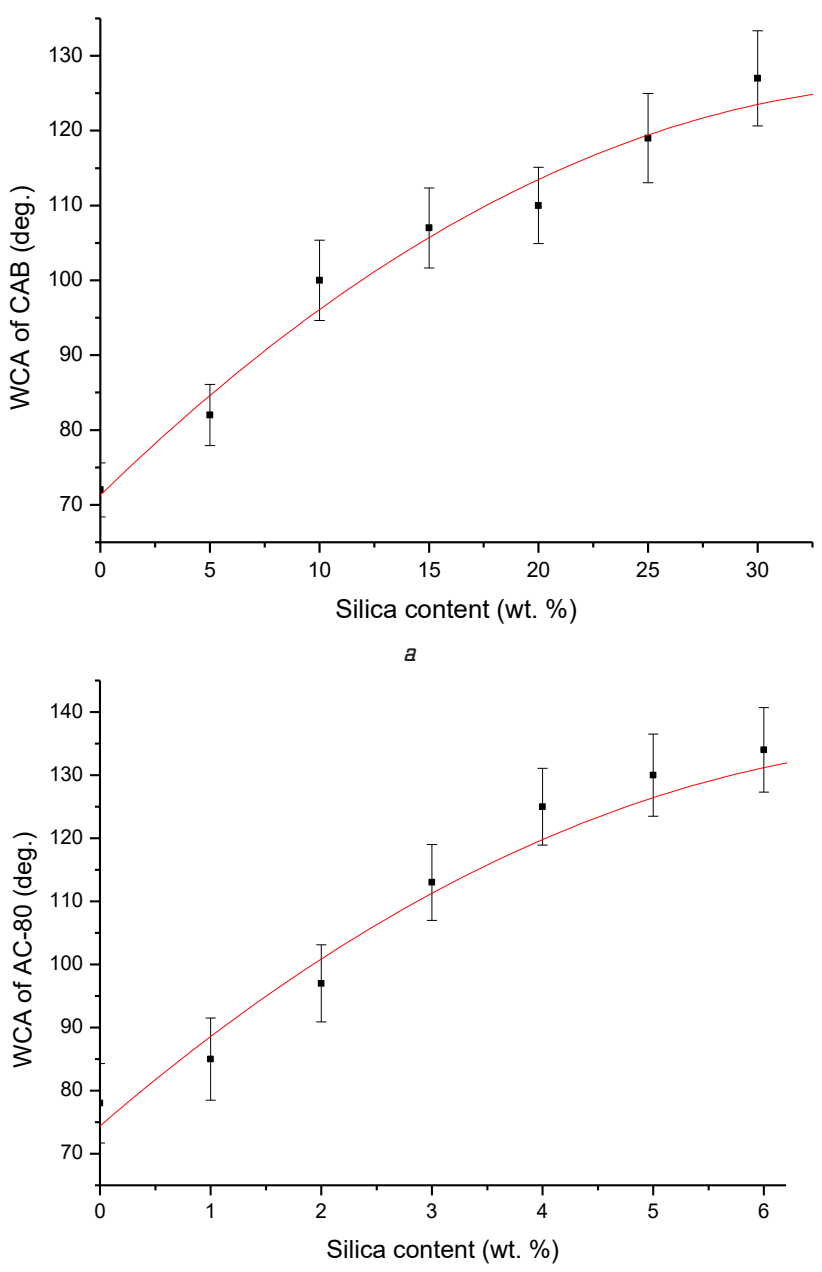

$b$

Fig. 1. Dependence of the angle of wetting with water WCA on the degree of filling with silica particles: a - cellulose acetobutyrate in the range from 0 to 30 wt. \%; $b$ - acrylic copolymer in the range from 0 to 6 wt. \%

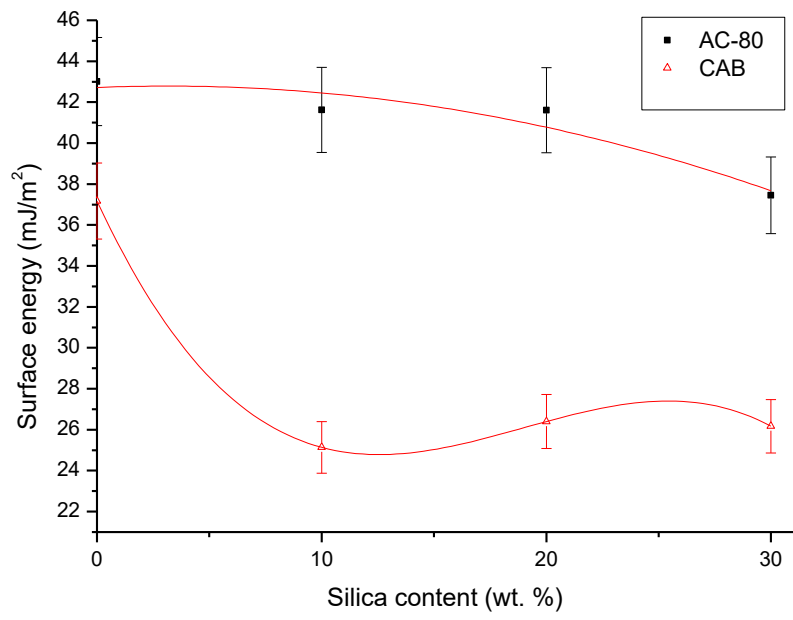

Fig. 2. Dependence of the surface energy of superhydrophobic coatings on the degree of filling with silicon dioxide in the range from 0 to 30 wt. \%: AC-80 - styrene-acrylic copolymer;

CAB - cellulose acetate butyrate
It is known that an increase in the contact angle is accompanied by a decrease in the surface energy of solids, which is explained by the stronger interaction between the molecules of the solid surface compared to the force of interaction between the solid and liquid phases [21]. Let's consider this process in more detail using the example of hydrophobization of acetate butyrate.

At first, a drop in energy is observed when adding a filler from 0 to $10 \mathrm{wt}$ \%, which is explained by the partial substitution of the polar groups of cellulose acetate by less polar groups of the aerosil hydrophobizator - dimethyldichlorosilane. Such changes in the chemical structure of the coating surface can explain the first pronounced increases in the angles of wetting with water in Fig. 1. Further addition of aerosil more than 10 wt. \% probably leads to the appearance of the first duels of nanoscale formations on the surface of the coating, accompanied by fluctuations in its surface energy. Introduction of 30 wt. \% modifier most likely provides stabilization of the surface structure of the coating, as a result of which it passes into a superhydrophobic state, as evidenced by the contact angle value of $127^{\circ}$. The identifier of the transition to the superhydrophobic state of the coating based on styrene-acrylic copolymer is the water contact angle of $134^{\circ}$.

The difference between the curves in Fig. 2 can be explained by the different crystallinity of the materials used, it can be noted in the analysis of photographs of the surface of untreated polymers obtained with an electron microscope (Fig. 3, a and Fig. 4, a). Thus, the surface of a cellulosic polymer (Fig. 3,a) is characterized by the presence of a globular structure, while an acrylic polymer (Fig. 4, $a$ ) is a pure transparent film, since the first material has an amorphous structure, and the second is crystalline [22, 23].

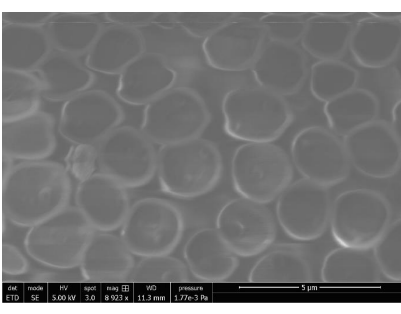

a

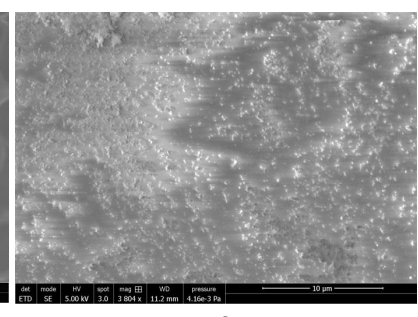

$b$
Fig. 3. SEM photo of coatings based on cellulose acetate butyrate: $a$ - without aerosil; $b$ - with the addition of 30 wt. \% aerosil

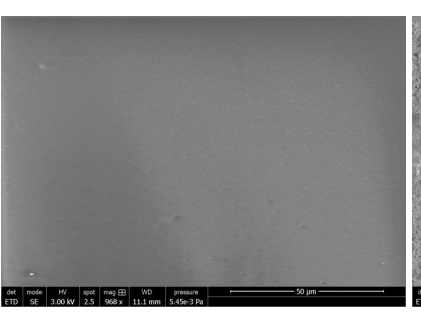

a

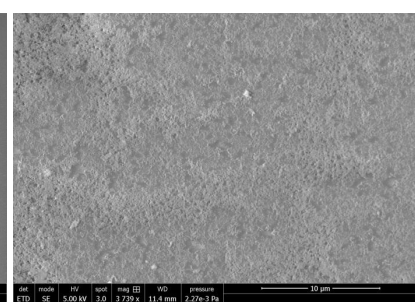

$b$
Fig. 4. SEM photo of coatings based on styrene-acrylic copolymer: $a$ - without aerosil; $b$ - with the addition of 6 wt. \% aerosil

Comparing the photos shown in Fig. 3, 4, it is possible to trace the change in the morphology of the coatings during the filling of aerosil film-formers, which in turn leads to an improvement in their water-repellent properties. The photographs in Fig. 3 show significant differences in the surfaces of cellulose coatings before $(a)$ and after $(b)$ hydrophobization. In the photo Fig. $3, a$ shows that only polymer 
molecules are present on the coating surface; the waterwetting angle of this coating is $72^{\circ}$. But the surface with the photo Fig. $3, b$ is dotted with nanoscale irregularities formed by particles of silicon dioxide, the contact angle of such a surface is $127^{\circ}$. In Fig. 4, similar changes are observed: the left side of photo $(a)$ is a flat film of styrene-acrylic copolymer without any pronounced irregularities, while the surface on the right side of photo $(b)$ has a clear roughness. The contact angles of conventional and superhydrophobic surfaces for comparison are $82^{\circ}$ and $134^{\circ}$, respectively.

\section{Conclusions}

It is shown that fumed silica is an effective means of regulating the texture of thin-layer coatings. It is found that the value of the effective concentration of hydrophobized silicon dioxide to obtain a superhydrophobic coating is 6 wt. \% for acrylic film former and 30 wt. \% for cellulose acetate butyrate. The angles of water wetting of coatings based on these film-formers reach $134^{\circ}$ and $127^{\circ}$, respectively.

Using the Owens-Wendt approach, it is shown that as the content of filler nanoparticles increases, the total surface energy of the material decreases. In the case of an acrylic film former, this decrease is sharper than in the case of acetobutyrate and amounts to $32 \%$ and $11 \%$, respectively.

\section{References}

1. Tóth, A., László, K. (2012). Chapter 5 - Water Adsorption by Carbons. Hydrophobicity and Hydrophilicity. Novel Carbon Adsorbent. Elsevier, 147-171. doi: http://doi.org/10.1016/ B978-0-08-097744-7.00005-3

2. Livey, D. T., Murray, P. (1956). Surface Energies of Solid Oxides and Carbides. Journal of the American Ceramic Society, 39 (11), 363-372. doi: http://doi.org/10.1111/j.1151-2916.1956.tb15606.x

3. Myronyuk, O., Dudko, V., Baklan, D., Melnyk, L. (2017). Study of structure influence on wear resistance of hierarchial superhydrophobic coatings. Eastern-European Journal of Enterprise Technologies, 3 (12 (87)), 44-49. doi: http://doi.org/ 10.15587/1729-4061.2017.103028

4. Myronyuk, O., Raks, V. A., Baklan, D., Vasyliev, G., Vanagas, E., Kurdil, N., Sivolapov, P. (2021). Water repellent coatings with hierarchal structures obtained on anodized aluminum with femtosecond laser ablation. Applied Nanoscience. doi: http:// doi.org/10.1007/s13204-021-01697-8

5. Shirtcliffe, N. J., McHale, G., I. Newton, M. (2011). The superhydrophobicity of polymer surfaces: Recent developments. Journal of Polymer Science Part B: Polymer Physics, 49 (17), 1203-1217. doi: http://doi.org/10.1002/polb.22286

6. Kim, J., Choi, S. (2018). 11 - Superhydrophobicity. Waterproof and water repellent textiles and clothing. Oxford: Woodhead Publishing, 267-297. doi: http://doi.org/10.1016/b978-0-08101212-3.00010-1

7. Wenzel, R. N. (1936). Resistance of solid surfaces to wetting by water. Industrial \& Engineering Chemistry, 28 (8), 988-994. doi: http://doi.org/10.1021/ie50320a024

8. Cassie, A. B. D., Baxter, S. (1944). Wettability of porous surfaces. Transactions of the Faraday Society, 40, 546-551. doi: http://doi.org/10.1039/tf9444000546

9. Bhushan, B., Nosonovsky, M. (2010). The rose petal effect and the modes of superhydrophobicity. Philosophical Transactions of the Royal Society A: Mathematical, Physical and Engineering Sciences, 368 (1929), 4713-4728. doi: http://doi.org/10.1098/ rsta.2010.0203

10. Rahmawan, Y., Moon, M.-W., Kim, K.-S., Lee, K.-R., Suh, K.-Y. (2009). Wrinkled, Dual-Scale Structures of Diamond-Like Carbon (DLC) for Superhydrophobicity. Langmuir, 26 (1), 484-491. doi: http://doi.org/10.1021/la902129k

11. Lu, H., Cai, R., Zhang, L.-Z., Lu, L., Zhang, L. (2020). Experimental investigation on deposition reduction of different types of dust on solar PV cells by self-cleaning coatings. Solar Energy, 206, 365-373. doi: http://doi.org/10.1016/j.solener.2020.06.012
12. Chen, H., Wang, F., Fan, H., Hong, R., Li, W. (2021). Construction of MOF-based superhydrophobic composite coating with excellent abrasion resistance and durability for self-cleaning, corrosion resistance, anti-icing, and loading-increasing research. Chemical Engineering Journal, 408, 127343. doi: http://doi.org/ 10.1016/j.cej.2020.127343

13. Li, P., Chen, X., Yang, G., Yu, L., Zhang, P. (2014). Preparation of silver-cuprous oxide/stearic acid composite coating with superhydrophobicity on copper substrate and evaluation of its friction-reducing and anticorrosion abilities. $A p$ plied Surface Science, 289, 21-26. doi: http://doi.org/10.1016/ j.apsusc.2013.10.068

14. Suryaprabha, T., Sethuraman, M. G. (2017). Design of electrically conductive superhydrophobic antibacterial cotton fabric through hierarchical architecture using bimetallic deposition. Journal of Alloys and Compounds, 724, 240-248. doi: http://doi.org/10.1016/j.jallcom.2017.07.009

15. Barthwal, S., Barthwal, S., Singh, B., Bahadur Singh, N. (2020). Multifunctional and fluorine-free superhydrophobic composite coating based on PDMS modified MWCNTs/ZnO with selfcleaning, oil-water separation, and flame retardant properties. Colloids and Surfaces A: Physicochemical and Engineering Aspects, 597, 124776. doi: http://doi.org/10.1016/j.colsurfa.2020.124776

16. Owens, D. K., Wendt, R. C. (1969). Estimation of the surface free energy of polymers. Journal of Applied Polymer Science, 13 (8), 1741-1747. doi: http://doi.org/10.1002/app.1969.070130815

17. Myronyuk, O. V., Prydatko, A. V., Sivolapov, P. V., Svidersky, V. A. (2014). Aspects of polymer surfaces wetting. EasternEuropean Journal of Enterprise Technologies, 1 (6 (67)), 23-26. doi: http://doi.org/10.15587/1729-4061.2014.20797

18. Van Oss, C. (2006). Interfacial Forces in Aqueous Media. Boca Raton: CRC Press. doi: http://doi.org/10.1201/9781420015768

19. Liu, W., Zhou, Z., Liao, X., Li, C., Tang, H., Xie, M. et. al. (2020). Tailoring ordered microporous structure of cellulosebased membranes through molecular hydrophobicity design. Carbohydrate Polymers, 229, 115425. doi: http://doi.org/10.1016/ j.carbpol.2019.115425

20. Ozbay, S., Erbil, H. Y. (2014). Solution copolymerization of perfluoroalkyl ethyl methacrylate with methyl methacrylate and butyl acrylate: Synthesis and surface properties. Colloids and Surfaces A: Physicochemical and Engineering Aspects, 452, 9-17. doi: http://doi.org/10.1016/j.colsurfa.2014.03.054

21. Shafrin, E. G., Zisman, W. A. (1960). Constitutive relations in the wetting of low energy surfaces and the theory of the retraction method of preparing monolayers. The Journal of Physical Chemistry, 64 (5), 519-524. doi: http://doi.org/10.1021/j100834a002

22. Wypych, G.; Wypych, G. (Ed.) (2016). CAB cellulose acetate butyrate. Handbook of Polymers. ChemTec Publishing, 39-42. doi: http://doi.org/10.1016/b978-1-895198-92-8.50013-6

23. Siljanovska Petreska, G., Auschra, C., Paulis, M. (2018). Confinement driven crystallization of ABA crystalline-soft-crystalline block copolymers synthesized via RAFT mediated miniemulsion polymerization. Polymer, 158, 327-337. doi: http://doi.org/ 10.1016/j.polymer.2018.10.073

Pavlo Sivolapov, Postgraduate Student, Department of Chemical Technology of Composition Materials, National Technical University of Ukraine «Igor Sikorsky Kyiv Polytechnic Institute», Kyiv, Ukraine, ORCID: https://orcid.org/0000-0001-7303-7179, e-mail: pavlo.sivolapov@gmail.com

Oleksiy Myronyuk, PhD, Associate Professor, Department of Chemical Technology of Composition Materials, National Technical University of Ukraine «Igor Sikorsky Kyiv Polytechnic Institute», Kyiv, Ukraine, ORCID: https://orcid.org/0000-0003-0499-9491, e-mail: o.myronyuk@kpi.ua

Denys Baklan, Postgraduate Student, Department of Chemical Technology of Composition Materials, National Technical University of Ukraine «Igor Sikorsky Kyiv Polytechnic Institute», Kyiv, Ukraine, ORCID: https://orcid.org/0000-0002-6608-0117, e-mail: d.baklan@kpi.ua

Taras Berehovyj, Department of Chemical Technology of Composition Materials, National Technical University of Ukraine «Igor Sikorsky Kyiv Polytechnic Institute», Kyiv, Ukraine, ORCID: https://orcid.org/ 0000-0001-7752-0758, e-mail: pro100taras.beregovoi@gmail.com 